\title{
The areolar strain concept applied to elasticity
}

\author{
I. D. Kotchergenko \\ Instituto Militar de Engenharia, Rio de Janeiro, Brazil
}

\begin{abstract}
In contrast to the books that start with solutions of Lamé-Navier equations using complex variables, the present article starts with a presentation of the fundamentals of the complex theory of two-dimensional elasticity. A new strain expression is derived and the compatibility conditions for these strains are given. The fundamental equations for the isotropic and orthotropic plane elasticity are also presented in somewhat new complex forms. The homogeneous equilibrium equation is presented in a complex form that proved to be easily solvable. Kolosov's general solution for the isotropic case is obtained in a fairly straightforward fashion. New equilibrium equations and boundary conditions for finite rotation are also given.
\end{abstract}

Keywords: areolar strain, compatibility equation, finite rotation, complex elasticity.

\section{Introduction}

The development of the theory of two-dimensional elasticity hereof is grounded on the concept of areolar strain. This concept was first presented by Kotchergenko [8], in 1983, though containing some errors. In this approach the strain is obtained by the division of two complex-valued quantities associated with $2 \mathrm{D}$ vectors and called areolar strain owing to the fact that its real part represents a radial strain and its imaginary part represents either a circumferential strain or a rotation. The quaternion concept can probably be used to extend the areolar strain concept to the 3D vectors case. Improvements on the linear theory were recently presented, Kotchergenko [9,10]. Equilibrium equations for finite rotation and several other improvements are now included. A detailed review of the main results obtained until now is provided in order to make this article self contained. 


\section{The areolar strain}

Let a region in the plane of the variables $z=x+i y$ and $\bar{z}=x-i y$ be mapped in a one-to-one manner onto the plane of the displacements $u(x, y)$ and $v(x, y)$ by means of the transformation $w(z, \bar{z})=u(x, y)+i v(x, y)$. Given the direction $\alpha$ of the vector $z-z_{0}$, where $z$ and $z_{0}$ are two neighboring points of the plane, the areolar strain is defined as the gradient of the vector field $w(z, \bar{z})$ in the direction $\alpha$, through the areolar derivative:

$$
\varepsilon=\lim _{z \rightarrow z_{0}} \frac{w-w_{0}}{z-z_{0}}=\frac{\frac{\partial w}{\partial z} d z+\frac{\partial w}{\partial \bar{z}} d \bar{z}}{d z} \quad \text { or } \quad \varepsilon=\frac{\partial w}{\partial z}+\frac{\partial w}{\partial \bar{z}} e^{-i 2 \alpha},
$$

where the polar form has been used for the ratio $\frac{d \bar{z}}{d z}=\frac{|d \bar{z}| e^{-i \alpha}}{|d z| e^{i \alpha}}=e^{-i 2 \alpha}$. This expression presupposes that $z$ tends to $z_{0}$, maintaining the direction $\alpha$. Since

$$
\begin{gathered}
2 \frac{\partial w}{\partial z}=\left(\frac{\partial u}{\partial x}+\frac{\partial v}{\partial y}\right)+i\left(\frac{\partial v}{\partial x}-\frac{\partial u}{\partial y}\right)=\theta+i 2 \omega \\
2 \frac{\partial w}{\partial \bar{z}}=\left(\frac{\partial u}{\partial x}-\frac{\partial v}{\partial y}\right)+i\left(\frac{\partial v}{\partial x}+\frac{\partial u}{\partial y}\right)=\xi+i \gamma
\end{gathered}
$$

the areolar strain can also be written in the form

$$
\varepsilon=1 / 2(\theta+i 2 \omega)+1 / 2(\xi+i \gamma) e^{-2 i \alpha} .
$$

When viewed in the polar form, the real part of the areolar strain represents a radial strain and the imaginary part represents either, a circumferential strain or a rotation. The second complex term is the complex shear strain. The components of the areolar strain are orthogonal as the quadratic form contained in the integrand of the work expression $U=1 / 2 \int C_{i j} \varepsilon_{i} \varepsilon_{j} d V$ can be converted into its canonic form

$$
U=1 / 2 \int\left[1 / 2\left(C_{11}+C_{12}\right) \theta^{2}+1 / 2\left(C_{11}-C_{12}\right) \xi^{2}+C_{13} \gamma^{2}\right] d V .
$$

\section{Compatibility equations}

If $z_{0}$ and $z$ are two points pertaining to the complex plane, their relative displacement is given by

$$
w-w_{0}=\int_{C} \varepsilon d z=\int_{C}\left(\frac{\partial w}{\partial z}+\frac{\partial w}{\partial \bar{z}} \frac{d \bar{z}}{d z}\right) d z=\int_{C} \frac{\partial w}{\partial z} d z+\frac{\partial w}{\partial \bar{z}} d \bar{z} .
$$

Since $w-w_{0}$ is independent of the path of integration $C$,

$$
d w=\frac{\partial w}{\partial z} d z+\frac{\partial w}{\partial \bar{z}} d \bar{z}
$$



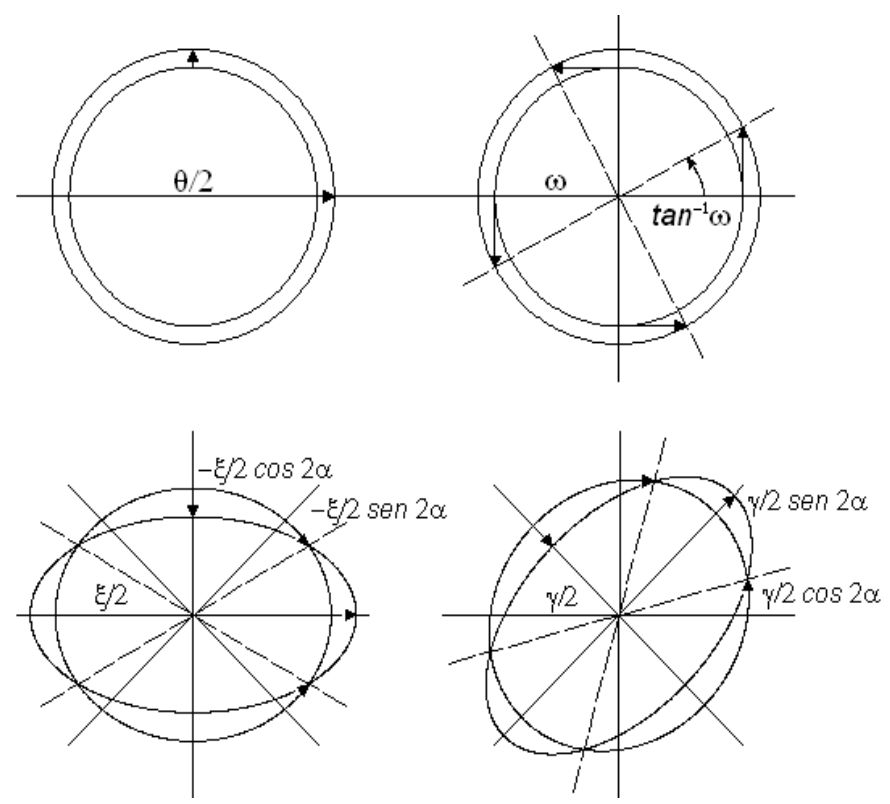

Figure 1: Fundamental modes of the areolar strain.

is a total differential. Consequentially, the displacement field must comply with the condition of continuity

$$
\frac{\partial}{\partial \bar{z}}\left(\frac{\partial w}{\partial z}\right)-\frac{\partial}{\partial z}\left(\frac{\partial w}{\partial \bar{z}}\right)=0
$$

Separating the real and imaginary parts of this equation, the following compatibility equations are obtained:

$$
\begin{aligned}
& \frac{\partial}{\partial x}(2 \omega-\gamma)+\frac{\partial}{\partial y}(\theta+\xi)=0, \\
& \frac{\partial}{\partial y}(2 \omega+\gamma)-\frac{\partial}{\partial x}(\theta-\xi)=0 .
\end{aligned}
$$

Saint-Venant's compatibility equation is obtained from these equations through elimination of the mode $\omega$, by applying a cross-differentiation followed by a subtraction. Saint-Venant's compatibility equation will then be satisfied for any rotational field, which may thus violate the compatibility conditions (8).

\section{Equilibrium equations}

For isotropic material, $1 / 2\left(C_{11}+C_{12}\right)=\lambda+\mu, 1 / 2\left(C_{11}-C_{12}\right)=\mu$ and $C_{13}=\mu$, thus the work expression given by eqn. (4) reduces to 


$$
U=1 / 2 \int\left[(\lambda+\mu) \theta^{2}+\mu\left(\xi^{2}+\gamma^{2}\right)\right] d V
$$

where $\lambda$ and $\mu$ are Lame's elastic material constants. The Euler equations for this functional are

$$
\begin{aligned}
& (\lambda+\mu) \frac{\partial \theta}{\partial x}+\mu\left(\frac{\partial \xi}{\partial x}+\frac{\partial \gamma}{\partial y}\right)=0 \\
& (\lambda+\mu) \frac{\partial \theta}{\partial y}+\mu\left(\frac{\partial \gamma}{\partial x}-\frac{\partial \xi}{\partial y}\right)=0
\end{aligned}
$$

Taking into account that

$$
\frac{\partial}{\partial z}(\xi+i \gamma)=\left(\frac{\partial \xi}{\partial x}+\frac{\partial \gamma}{\partial y}\right)+i\left(\frac{\partial \gamma}{\partial x}-\frac{\partial \xi}{\partial y}\right)=\Delta u(x, y)+i \Delta v(x, y)
$$

where the symbol $\Delta$ stands for the Laplacian operator, Lamé's homogeneous equilibrium equations can be presented in the following complex form:

$$
2 \frac{\partial}{\partial \bar{z}}[(\lambda+\mu) \theta]+2 \frac{\partial}{\partial z}[\mu(\xi+i \gamma)]=0
$$

Multiplying each term of eqn (7) by a different complex elastic constant will result in an equation in terms of stresses. For isotropic material, undergoing small strain and small rotations, the operations required for obtaining Lame's homogeneous equilibrium equation are

$$
\frac{\partial}{\partial \bar{z}}\left[(\lambda+\mu)\left(2 \frac{\partial w}{\partial z}-i \omega\right)\right]-\frac{\partial}{\partial z}\left(\frac{\lambda}{2} 2 \frac{\partial w}{\partial \bar{z}}\right)=0 .
$$

Observe that only one out of the two $i \omega$, which appear in the term $i 2 \omega$, was removed. The term $i \omega$ represents a local rotation. Taking the derivatives of $\omega$ from eqns. (8) and substituting into eqn. (12) will give eqn. (11)

\section{Boundary conditions}

Applying Green's formula (e.g. Courant and Hilbert [1]) to eqn. (11), in the complex form,

$$
2 \iint_{\Omega}\left\{\frac{\partial}{\partial \bar{z}}[(\lambda+\mu) \theta]+\frac{\partial}{\partial z}[\mu(\xi+i \gamma)]\right\} d x d y=\frac{1}{i} \oint_{C}(\lambda+\mu) \theta d z-\mu(\xi+i \gamma) d \bar{z},
$$

the traction vector on a boundary curve $C$ results

$$
T=(\lambda+\mu) \theta-\mu(\xi+i \gamma) e^{-2 i \alpha},
$$

where $\alpha$ points towards the direction of the vector element of arc $d z$ of the closed contour curve $C$. Observe that if $n$ the unit outward vector, normal to the element of $\operatorname{arc} d s=|d z|$, then $n d s=-i d z=1 / i d z$. 


\section{Other forms for the equilibrium equations}

The compatibility equations (7) can also be rewritten in the following complex form

$$
\frac{\partial}{\partial \bar{z}}(\theta+i 2 \omega)-\frac{\partial}{\partial z}(\xi+i \gamma)=0
$$

Equation (11) can hence be reduced to the following holomorphic function (Love, [4]),

$$
\frac{\partial}{\partial \bar{z}}[\theta+i \eta 2 \omega]=0
$$

where $\eta=\frac{\mu}{\lambda+2 \mu}$. The modes $\theta$ and $\omega$ are hence harmonic functions.

The writer succeeded in rewriting the equilibrium equation in another form that proved to be easily solvable. The elimination of the derivatives of $\theta$ between equations (8) and (16), gives

$$
\begin{aligned}
& 2 \frac{\partial \omega}{\partial x}=\frac{\lambda+2 \mu}{\lambda+\mu}\left(\frac{\partial \gamma}{\partial x}-\frac{\partial \xi}{\partial y}\right), \\
& 2 \frac{\partial \omega}{\partial y}=-\frac{\lambda+2 \mu}{\lambda+\mu}\left(\frac{\partial \xi}{\partial x}+\frac{\partial \gamma}{\partial y}\right) .
\end{aligned}
$$

The elimination of the derivatives of $\omega$, from the same pair of equations, gives

$$
\begin{aligned}
& \frac{\partial \theta}{\partial x}=-\frac{\mu}{\lambda+\mu}\left(\frac{\partial \xi}{\partial x}+\frac{\partial \gamma}{\partial y}\right), \\
& \frac{\partial \theta}{\partial y}=-\frac{\mu}{\lambda+\mu}\left(\frac{\partial \gamma}{\partial x}-\frac{\partial \xi}{\partial y}\right) .
\end{aligned}
$$

Combination of these equations yields:

$$
2 \frac{\partial}{\partial z}(\theta+i 2 \omega)=-\chi\left[\left(\frac{\partial \xi}{\partial x}+\frac{\partial \gamma}{\partial y}\right)-i\left(\frac{\partial \gamma}{\partial x}-\frac{\partial \xi}{\partial y}\right)\right],
$$

Or after eqns. (2),

$$
\frac{\partial^{2} w}{\partial z^{2}}+\chi \frac{\partial^{2} w}{\partial z \partial \bar{z}}=0
$$

where $\chi=\frac{\lambda+3 \mu}{\lambda+\mu}$ for plane strain and $\chi=\frac{\lambda^{*}+3 \mu}{\lambda^{*}+\mu}$, for plane stress, observing that $\lambda^{*}=\frac{2 \lambda \mu}{\lambda+2 \mu}$.

\section{General solution}

Differentiation of eqn. (11) in $z$ results 
584 Computational Methods and Experimental Measurements XIII

$$
2(\lambda+\mu) \frac{\partial^{2} \theta}{\partial z \partial \bar{z}}+2 \mu \frac{\partial^{2}}{\partial z^{2}}[(\xi+i \gamma)]=0
$$

Since $4 \frac{\partial^{2}}{\partial z \partial \bar{z}}$ is the Laplacian operator and $\theta$ is a harmonic function, this equation reduces to

$$
2 \mu \frac{\partial^{2}}{\partial z^{2}}[(\xi+i \gamma)]=0
$$

or after eqns. (2), to

$$
2 \frac{\partial}{\partial z}\left(\frac{\partial^{2} w}{\partial z \partial \bar{z}}\right)=0
$$

Integration in $z$ gives

$$
\frac{\partial^{2} w}{\partial z \partial \bar{z}}=-\frac{1}{2 \mu} \overline{\varphi^{\prime \prime}(z)},
$$

where $\overline{\varphi^{\prime \prime}(z)}$ is the conjugate of an analytic function. Integrating eqn. (23) in $z$, results

$$
\frac{\partial w}{\partial \bar{z}}=\frac{1}{2}(\xi+i \gamma)=-\frac{1}{2 \mu}\left[z \overline{\varphi^{\prime \prime}(z)}+\overline{\psi^{\prime}(z)}\right]
$$

Equations (20) and (23) furnish

$$
\frac{\partial^{2} w}{\partial z^{2}}=\frac{\chi}{2 \mu} \varphi^{\prime \prime}(z),
$$

which after integration in $z$, results

$$
\frac{\partial w}{\partial z}=\frac{\chi}{2 \mu} \varphi^{\prime}(z)+\zeta^{\prime}(\bar{z}) \cdot
$$

Differentiation of this equation with respect to $\bar{z}$ yields

$$
\frac{\partial}{\partial \bar{z}}\left(\frac{\partial w}{d z}\right)=\zeta^{\prime \prime}(\bar{z})
$$

From eqn. (23), results $\zeta^{\prime \prime}(\bar{z})=-\frac{1}{2 \mu} \overline{\varphi^{\prime \prime}(z)}$ and hence

$$
\zeta^{\prime}(\bar{z})=-\frac{1}{2 \mu} \overline{\varphi^{\prime}(z)}+c,
$$

where $c$ is a complex constant. Substitution into eqn. (25) yields

$$
\frac{\partial w}{\partial z}=\frac{1}{2}(\theta+i 2 \omega)=\frac{1}{2 \mu}\left[\chi \varphi^{\prime}(z)-\overline{\varphi^{\prime}(z)}\right]+\frac{1}{2}\left(\theta_{0}+i 2 \omega_{0}\right) .
$$

Using eqns. (28) and (24), the integration of the total differential, eqn. (5), gives Kolosov-Muskhelishvili's general solution, [2,3,5,7]:

$$
w=w_{0}+\frac{1}{2}\left(\theta_{0}+i 2 \omega_{0}\right) z+\frac{1}{2 \mu}\left[\chi \varphi(z)-z \overline{\varphi^{\prime}(z)}-\overline{\psi(z)}\right] .
$$


The addition of Eqn (28) to its conjugate gives

$$
\theta=\frac{\chi-1}{2 \mu}\left[\varphi^{\prime}(z)+\overline{\varphi^{\prime}(z)}\right]+\theta_{0} .
$$

The subtraction of Eqn (28) from its conjugate gives

$$
\omega=\frac{\chi+1}{2 \mu}\left[\frac{\varphi^{\prime}(z)-\overline{\varphi^{\prime}(z)}}{2 i}\right]+\omega_{0} .
$$

Kolosov's formulas, for stresses are obtained directly from eqns. (30) and (24):

$$
\begin{gathered}
\sigma_{11}+\sigma_{22}=2\left[\varphi^{\prime}(z)+\overline{\varphi^{\prime}(z)}\right]+2(\lambda+\mu) \theta_{0}, \\
\sigma_{11}-\sigma_{22}+i 2 \sigma_{12}=-2\left[z \overline{\varphi^{\prime \prime}(z)}+\overline{\psi^{\prime}(z)}\right] .
\end{gathered}
$$

The areolar strain, in terms of analytic functions assumes the form

$$
\varepsilon=\frac{1}{2}\left(\theta_{0}+i 2 \omega_{0}\right)+\frac{1}{2 \mu}\left[\chi \varphi^{\prime}(z)-\overline{\varphi^{\prime}(z)}\right]-\frac{1}{2 \mu}\left[z \overline{\varphi^{\prime \prime}(z)}+\overline{\psi^{\prime}(z)}\right] e^{-i 2 \alpha} .
$$

\section{Equilibrium equations for an orthotropic plane}

The writer explored the possibility of applying to the orthotropic plane case the same approach used with eqn. (11). Taking the compatibility equation in the form of eqn. (15) and removing one out of the two $i \omega$ present into the term $i 2 \omega$; then multiplying each complex term of this equation by different complex elastic constants, will result in the following equation in terms of stresses:

$$
\left(a_{1}+i a_{2}\right) \frac{\partial}{\partial z}(\theta+i \omega)-\left(b_{1}+i b_{2}\right) \frac{\partial}{\partial z}(\xi+i \gamma)=0 .
$$

Substitution of the derivatives of the rotation, obtained from eqn. (8), gives

$$
\left(a_{1}+i a_{2}\right) \frac{\partial \theta}{\partial \bar{z}}+\left[\left(a_{1}+i a_{2}\right)-2\left(b_{1}+i b_{2}\right)\right] \frac{\partial}{\partial z}(\xi+i \gamma)=0 .
$$

Lamé's equilibrium equations are obtained letting $a_{2}=0, b_{2}=0, a_{1}=\lambda+\mu$ and $b_{1}=\lambda / 2$.

Application of Green's formula to eqn. (36), gives the following generalization of the traction vector formula, previously depicted in eqn. (14):

$$
T=\left(a_{1}+i a_{2}\right) \theta-\left[\left(a_{1}+i a_{2}\right)-2\left(b_{1}+i b_{2}\right)\right](\xi+i \gamma) e^{-i 2 \alpha}
$$

Using the same procedure as the one used for obtaining eqn. (16) leads to the same Love's form of holomorphic function:

$$
\frac{\partial}{\partial \bar{z}}[\theta+i \eta 2 \omega]=0,
$$

where

$$
\eta=\frac{\left(a_{1}-2 b_{1}\right)+i\left(a_{2}-2 b_{2}\right)}{2\left[\left(a_{1}-b_{1}\right)+i\left(a_{2}-b_{2}\right)\right]} .
$$

Observe that for the isotropic plane, $\eta$ will be reduced to a real constant. 
Separating the real and imaginary parts yields:

with $\eta=\eta_{1}+i \eta_{2}$, where

$$
\begin{aligned}
& \frac{\partial \theta}{\partial x}=\eta_{1} 2 \frac{\partial \omega}{\partial y}+\eta_{2} 2 \frac{\partial \omega}{\partial x}, \\
& \frac{\partial \theta}{\partial y}=-\eta_{1} 2 \frac{\partial \omega}{\partial x}+\eta_{2} 2 \frac{\partial \omega}{\partial y},
\end{aligned}
$$

$$
\begin{gathered}
\eta_{1}=\frac{a_{1}^{2}+a_{2}^{2}-3\left(a_{1} b_{1}+a_{2} b_{2}\right)+2\left(b_{1}^{2}+b_{2}^{2}\right)}{2\left[\left(a_{1}-b_{1}\right)^{2}+\left(a_{2}-b_{2}\right)^{2}\right]}, \\
\eta_{2}=\frac{a_{2} b_{1}-a_{1} b_{2}}{2\left[\left(a_{1}-b_{1}\right)^{2}+\left(a_{2}-b_{2}\right)^{2}\right]} .
\end{gathered}
$$

Differentiations of eqns. (40), yields

$$
\frac{\partial^{2} \theta}{\partial x^{2}}+\frac{\partial^{2} \theta}{\partial y^{2}}=\eta_{2} 2\left(\frac{\partial^{2} \omega}{\partial x^{2}}+\frac{\partial^{2} \omega}{\partial y^{2}}\right)
$$

Other differentiations of eqns. (40), yields additionally:

$$
\frac{\partial^{2} \omega}{\partial x^{2}}=\frac{1}{2 \eta_{1}}\left(\eta_{2} 2 \frac{\partial^{2} \omega}{\partial x \partial y}-\frac{\partial^{2} \theta}{\partial x \partial y}\right) \quad \text { and } \quad \frac{\partial^{2} \omega}{\partial y^{2}}=\frac{1}{2 \eta_{1}}\left(-\eta_{2} 2 \frac{\partial^{2} \omega}{\partial x \partial y}+\frac{\partial^{2} \theta}{\partial x \partial y}\right) .
$$

Hence $\frac{\partial^{2} \omega}{\partial x^{2}}+\frac{\partial^{2} \omega}{\partial y^{2}}=0$, and after eqn. (42), both $\omega$ and $\theta$ remain harmonics also for the orthotropic plane case.

\section{Finite rotations}

The gain of area during a plane deformation is given by

$$
d A=\theta+\frac{\theta^{2}}{4}+\omega^{2}-\frac{1}{4}\left(\xi^{2}+\gamma^{2}\right)=\theta+\left|\frac{\partial w}{d z}\right|^{2}-\left|\frac{\partial w}{\partial \bar{z}}\right|^{2},
$$

which is obtained from the determinant

$$
1+d A=\left[\begin{array}{cc}
1+\frac{\partial u}{\partial x} & \frac{\partial u}{\partial y} \\
\frac{\partial v}{\partial x} & 1+\frac{\partial v}{\partial y}
\end{array}\right]
$$

Observing fig. 1, it can be seen that a finite "rotation" $\omega$ has a significant influence into the change of area of a plane elastic body. In some bending and buckling problems, it is admitted to disregard strain terms squared, except for $\omega^{2}$, reducing $d A$ to

$$
d A=\theta+\omega^{2}
$$


A rigid body rotation in this case can be approximated by the condition $\theta+\omega^{2}=0$. Then, for a point to describe a circular path of $\operatorname{arc} \Omega=\tan ^{-1} \omega$, it is required that the expansion produced by the mode $\omega$ be neutralized by the shrinkage $\theta=-\omega^{2}$. For the condition given by eqn. (45), the work expression assumes the form

$$
U=1 / 2 \int(\lambda+\mu)\left(\theta+\omega^{2}\right)^{2}+\mu\left(\xi^{2}+\gamma^{2}\right) d x d y .
$$

The Euler equations for this functional are

$$
\begin{gathered}
(\lambda+\mu) \frac{\partial \theta}{\partial x}+\mu\left(\frac{\partial \xi}{\partial x}+\frac{\partial \gamma}{\partial y}\right)+(\lambda+\mu)\left[-\frac{\partial}{\partial y}(\omega \theta)+\frac{\partial}{\partial x}\left(\omega^{2}\right)-3 \omega^{2} \frac{\partial \omega}{\partial y}\right]=0 \\
(\lambda+\mu) \frac{\partial \theta}{\partial y}+\mu\left(\frac{\partial \gamma}{\partial x}-\frac{\partial \xi}{\partial y}\right)+(\lambda+\mu)\left[\frac{\partial}{\partial x}(\omega \theta)+\frac{\partial}{\partial y}\left(\omega^{2}\right)+3 \omega^{2} \frac{\partial \omega}{\partial x}\right]=0 .
\end{gathered}
$$

This system of equations can be written in the complex form

$$
(\lambda+\mu) \frac{\partial}{\partial \bar{z}}\left[(1+i \omega)\left(\theta+\omega^{2}\right]+\mu \frac{\partial}{\partial z}(\xi+i \gamma)=0 .\right.
$$

Applying Green's formula, the traction vector on a closed curve $C$, in the undeformed reference frame, results

$$
T=(\lambda+\mu)\left[(1+i \omega)\left(\theta+\omega^{2}\right)\right]-\mu(\xi+i \gamma) e^{-i 2 \alpha} .
$$

Accordingly with eqns. (I.22) from Novozhilov [6], the shearing modes due to finite strains are: $\hat{\xi}=\xi+\frac{1}{2} \theta \xi+\omega \gamma$ and $\hat{\gamma}=\gamma+\frac{1}{2} \theta \gamma-\omega \xi$, hence discarding the strains squared, other than those containing $\omega$, results $\xi=\hat{\xi}-\omega \gamma$ and $\gamma=\hat{\gamma}+\omega \xi$. If the following approximations are used accordingly:

$$
\theta+\omega^{2}=\frac{\sigma_{x}+\sigma_{y}}{2(\lambda+\mu)}, \quad \xi=\frac{\sigma_{x}-\sigma_{y}}{2 \mu}-\omega \frac{\tau}{\mu} \text { and } \gamma=\frac{\tau}{\mu}+\omega \frac{\sigma_{x}-\sigma_{y}}{2 \mu},
$$

the equilibrium equations (47) in terms of stresses, referred to the undeformed reference frame, become

$$
\begin{aligned}
& \frac{\partial \sigma_{x}}{\partial x}+\frac{\partial \tau}{\partial y}-\frac{\partial}{\partial y}\left(\omega \sigma_{y}\right)-\frac{\partial}{\partial x}(\omega \tau)=0, \\
& \frac{\partial \sigma_{y}}{\partial y}+\frac{\partial \tau}{\partial x}+\frac{\partial}{\partial x}\left(\omega \sigma_{x}\right)+\frac{\partial}{\partial y}(\omega \tau)=0 .
\end{aligned}
$$

These are exactly the equilibrium equations (II.49) given by Novozhilov, [6].

\section{References}

[1] Courant, R. \& Hilbert, D., Methods of Mathematical Physics, Vol. II, Wiley: New York, pp. 350-351, 1989. 
[2] England, A. H., Complex Variable Methods in Elasticity, Wiley, pp. 2849, 1971.

[3] Kalandiya, A. I., Mathematical Methods of Two-Dimensional Elasticity, Mir Publishers, pp. 307-339, 1975.

[4] Love, A.E.H., A Treatise on the Mathematical Theory of Elasticity, Cambridge University Press, pp. 204-220, 1927.

[5] Muskhelishvili, N.I., Some Basic Problems of the Theory of Elasticity, Noordhoff, Groningen, 1963.

[6] Novozhilov, V.V., Foundations of the Nonlinear Theory of Elasticity, Dover Publications: Mineola, New York, pp. 83-84, 1999.

[7] Sokolnikoff, I.S., Mathematical Theory of Elasticity, McGraw-Hill, 1956.

[8] Kotchergenko, I.D., Unsymmetrical Plane Elasticity, Recent Advances in Engineering Mechanics, Proceedings of the Fourth Engineering Mechanics Division Specialty Conference, ASCE, eds. W.F. Chen \& A.D.M. Lewis, Vol. I, pp. 385-388, 1983.

[9] Kotchergenko, I.D., Kolosov-Mushkhelishvili Formulas Revisited, $11^{\text {th }}$ International Conference on Fracture, Turin, March 2005.

[10] Kotchergenko, I.D., Applications of Generalized Analytic Functions to Elasticity, CMM-2005-Computer Methods in Mechanics, Polish Academy of Sciences, June 2005. 